\title{
Coming soon: nowhere to hide for hospital managers
}

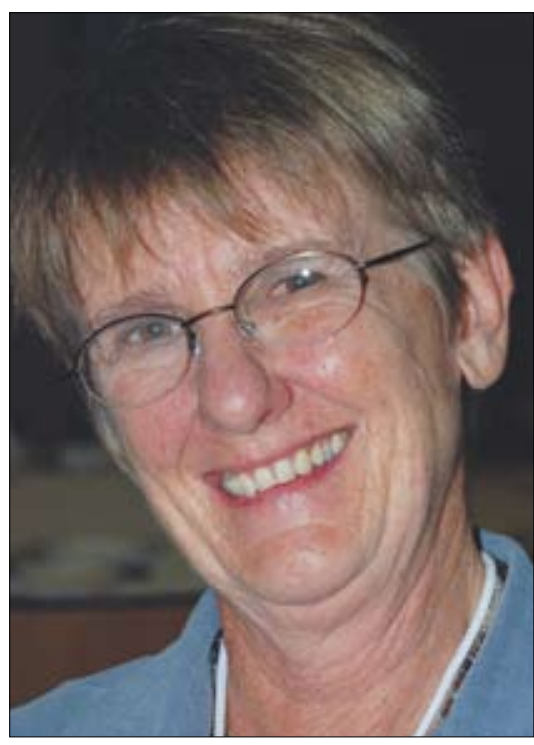

Dr Carol Marshal, Director of the Office of Healthcare Standards Compliance in the National Department of Health.

\section{Picture: Chris Bateman}

Hospital and health care managers and administrators will in future be measured against mandatory core standards and judged on outcomes, with little place to hide and less room to shift responsibility, the country's new standards certification chief warned last month.

Dr Carol Marshal, Director of the Office of Healthcare Standards Compliance (OHSC), in the national Department of Health has the full backing of her Director General, Precious Matsotso. This emerged in a speech the pair co-wrote for a health care safety conference in Cape Town last month. It became clear from the speech, delivered on Matsoso's behalf by Marshal and in a later interview with Marshal, that the 'slap on the wrist' approach - driven by a plethora of patient safety policies/guidelines and the absence of a uniform, credible means of probing health care delivery problems could soon be history.

She and her start-up team of 20 have spent nearly three years criss-crossing the country, consulting patient safety experts locally and overseas to set up national core standards for both private and public health establishments in advance of the National Health Insurance (being targeted for next year). Last month they briefed provincial leaders on how to use the new measurement tools for these core standards, so that once the National Health Amendment Bill is passed making the OHSC a statutory regulatory body, there can be no easy excuses. National Health Minister, Dr Aaron Motsoaledi, wants Marshal's office legally functioning by September this year, but is dependent on Parliament first passing the bill, which may take longer.

\section{Accountable at last?}

It will be the first time local public hospital and provincial managers are legally (besides the millions of taxpayer rands paid out annually for patient liability claims) called to account for health care outcomes at their facilities. In the speech Matsoso said government was 'deeply concerned' by dissatisfied patients complaining of rude and uncaring staff, dirty and unsafe hospitals and clinics and of waiting in long queues when seriously ill, often returning home without their medicine or any treatment.

'We have excellent policies and guidelines but we don't implement them, neither at the level of management nor even at the level of patient care,' she admitted. Poor quality, poor reliability in following best practices and a lack of accountability for poor results persisted - aggravated by responsibility being diluted 'among many different people at different levels of the system'.

\section{'We have excellent policies and} guidelines but we don't implement them, neither at the level of management nor even at the level of patient care,' she admitted.

'When there are no consequences, where management is found wanting, why should managers make an added effort?' she asked, adding that conversely, those who gave of their best every day were seldom recognised. Health Minister, Dr Aaron Motsoaledi had signed a service delivery agreement for his sector, reflecting a commitment to improving the health of mothers and children, life expectancy through dealing proactively with HIV/ AIDS, TB and chronic non-communicable diseases. The entire health system was being re-engineered towards a more preventive and primary health care approach while Marshal's outfit had synthesised existing regulations, policies, guidelines and protocols for betterquality health care.
This set of core standards would serve as a screening tool to identify where the outcome was 'problematic', while a set of measurement tools would measure and rate the levels of compliance. 'We need to close the policy implementation gap, Matsoso said.

\section{Facility governance in a 'sad state' - Matsoso}

She added that until now the flouting of policies, protocols and guidelines by health professionals had also contributed to notoriously poor outcomes at facilities. While a high patient load was often cited for the decline in standards of safety and care, the absence of clear clinical and corporate governance perpetuated 'a sad state' of affairs, which she put down to 'weak leadership and ethics'. In future clinical teams would have clearly defined roles and responsibilities with measured outcomes 'to improve ownership and accountability', while reporting and team discussion of errors or risky situations would be encouraged. Knowing that they could be subject to an external probe identifying clinical and systemic failings would hopefully 'serve as an added incentive' for health care professionals. Marshal's office would combine the functions of an inspectorate with an ombudsman's office that would probe serious complaints and systemic barriers to safe, quality care.

\section{Knowing that they could be} subject to an external probe identifying clinical and systemic failings would hopefully 'serve as an added incentive' for health care professionals.

An early-warning system with an as yet to be identified set of indicators or incidents would be predicated on compulsory data reporting to the OHSC, leading to an ad hoc inspection or probe. Marshal elaborated on this, citing the all too common example of a hospital with an alarmingly elevated maternal mortality rate.

'There can only be three things wrong: the data are incorrect, there's a serious problem in the community, or there's a serious problem in the hospital - so we send in a team. That is really exciting to me, but obviously it will take years to set up; this is no overnight wonder.' 
Get the basics right - Marshal She said that, simply put, her office had three methods of evaluating a facility: compliance with standards, the 'patient voice' through complaints and an ombudsman, and outcome indicators with an early-warning system. Honing in on a World Health Organization (WHO) report that more than half of compliance gaps could be solved at facility level without further intervention or funding, she cited the North West Province. Here the OHSC had hired external expertise to help one provincial hospital investigate why it had such long pharmacy queues and in one week cut them down by $60 \%$ - rolling out the methodology to other North West hospitals with similar results. The successes were now being replicated across the country.

'Our knee-jerk reaction in South Africa is to throw more money at it - the examples are legion - but if people just do what they're supposed to do and avoid all these unnecessary bureaucratic steps ... we really don't have to make life difficult for ourselves!'

\section{Pressed on the often counter- productive result of a punitive approach towards quality improvement, Marshal agreed but countered: 'Our minister has been very clear there has to be some accountability. You can't just say "Dear, Dear ... you're doing your best under very difficult circumstances".'}

Pressed on the often counter-productive result of a punitive approach towards quality improvement, Marshal agreed but countered: 'Our minister has been very clear there has to be some accountability. You can't just say "Dear, Dear ... you're doing your best under very difficult circumstances". Yes, sometimes the system is at fault. Everybody agrees that no-blame is great, but where we are in South Africa right now you can't do that. A hospital is not an airport or restaurant; it requires specialist knowledge and provisions. If you're the CEO of a hospital you have to understand that you cannot save money by not buying soap - that's simply unacceptable. No-blame has its limits!'

The context she is talking into includes faulty, outdated equipment, chronic health care staffing shortages leading to impossibly high patient/nurse, doctor/patient ratios, long working hours, an inappropriate skills mix, a huge HIV/AIDS/TB driven burden of disease, dismal waste management and infection control and poor stewardship/ leadership, to mention a few.

The OHSC will have a R28 million budget during its first official year (probably 2012) and R48 million the following year, after which this will drop slightly, the Bill stating that Marshal's office must then raise its own revenues by charging for services. 'Basically this will probably mean charging for inspections and/or the issuing of certificates,' Marshal explained.

The OHSC has until the end of this year to review its tools and methodology on facility assessment and will begin with public hospitals. Private hospitals have yet to be formally consulted on the process (although a draft measurement tool for them exists), but have volunteered their staff expertise to Marshal, who is keen to 'share knowledge'.

\section{Winging it alone or consulting widely?}

Asked whether she and the 16-year-old local NGO, the Council for Health Service Accreditation of Southern Africa(COHSASA), had mended fences following a fallout over measurement tools, Marshal replied tersely, 'Yes we're talking - we always have.'
COHSASA is the most experienced local accreditation body among those who tendered internationally for a multi-million rand OHSC contract to 'refine national core standards plans and guidelines and build implementation capacity in provinces. The contract was suddenly withdrawn without explanation last month. In March last year COHSASA broke off contact with the OHSC after two years of providing ad hoc advice, the bone of contention being the efficacy of the OHSC's standards of measurement and training. The OHSC turned to the United Kingdom's Care Quality Commission to audit its tools and processes. Marshal said that for an accreditation body to last, the responsibility had to be 'owned by government', adding, 'government cannot abrogate its' responsibility.

The Safecare Initiative, at whose founding conference Marshal spoke, is an international quality framework aimed at developing and applying universal standards of health care service delivery in resource-restricted settings and providing subsidised health care financing mechanisms. Three bodies, COHSASA, the United States-based Joint Commission International (JCI) and the PharmAccess Foundation of the Netherlands, have combined their resources and skills to do this, beginning in sub-Saharan Africa.

Marshal described the expert inputs at the conference as 'fascinating - obviously we'll tap into whatever expertise we can find, including the methodologies in scoring and reporting. Their information systems are beautiful and the technology is brilliant and the measurement expertise is obviously valuable - but they didn't talk about the sustainability of the initiative'.

\section{Chris Bateman}

1. Bateman C. Government crafts its own hospital quality standards, sans world class local body. S Afr Med J 2010;100:620-623. 